\title{
Tratamento eletroquímico de efluentes industriais - alternativa para a remoção de contaminantes e potencial aproveitamento de $\mathrm{H}_{2}$
}

\section{Electrochemical treatment of industrial effluents - alternative for contaminants removal and $\mathrm{H}_{2}$ obtainment}

Laisa Cristina Candido Maia ${ }^{1}$, Hermano Cezar Medaber Jambo ${ }^{2}$, José Antonio da Cunha Ponciano Gomes ${ }^{1}$

\author{
${ }^{1}$ Laboratório de Corrosão - Labcorr - PEMM/COPPE/UFRJ Caixa Postal: 68505 - 21941-914, Rio de Janeiro, RJ \\ e-mail: laisa@metalmat.ufrj.br \\ e-mail: ponciano@metalmat.ufrj.br \\ ${ }^{2}$ Petróleo Brasileiro SA - Avenida Chile, 65 - 20031-912, Rio de Janeiro, RJ \\ e-mail: cezarmedaber@gmail.com
}

\section{RESUMO}

A utilização de tratamentos eletroquímicos como recurso tecnológico alternativo para o tratamento de efluentes industriais tem atraído considerável atenção, apresentando características vantajosas como facilidade de automação, alta eficiência e versatilidade em menor tempo de tratamento, além de requerer menor espaço físico para suas instalações. A diminuição do uso de produtos químicos para o tratamento, uma vez que o elétron é o principal reagente no processo, e a possibilidade de reaproveitamento de coprodutos gerados também se configuram em importantes oportunidades dentro do atual cenário global de sustentabilidade e energia. Essas tecnologias comportam ainda o uso de fontes renováveis de energia. Um coproduto catódico de grande interesse ambiental e econômico é o hidrogênio, potencialmente coletado e armazenado.

Nesse contexto, os materiais são de importância fundamental, pois a eficiência e sustentabilidade dos processos eletroquímicos dependem da utilização de materiais com propriedades como baixa impedância eletroquímica e elevada resistência à corrosão, além de elevada atividade para a oxidação dos compostos de interesse, em especial os materiais selecionados para as etapas anódicas.

O objetivo deste artigo é apresentar, em linhas gerais, um dos trabalhos desenvolvidos no Labcorr voltados para o tratamento eletroquímico de efluentes industriais visando a remoção de $\mathrm{N}_{-} \mathrm{NH}_{3}$ e DQO. Técnicas eletroquímicas foram utilizadas para a seleção dos materiais usados como anodos e catodos. Configurações para utilização de $\mathrm{NaOCl}$ como oxidante indireto no processo foram avaliadas. Os resultados mostraram que um reator de placas paralelas com anodos DSA e catodos de aço carbono e injeções de $\mathrm{NaOCl}$ ao início do tratamento e em ponto intermediário do processo foram efetivos na remoção dos compostos desejados, indicando ainda boa oportunidade para o reaproveitamento de produtos como nitrito e hidrogênio, o primeiro como inibidor de corrosão e o segundo podendo ser utilizado como fonte de energia para alimentar o próprio sistema de tratamento de forma semiautônoma.

Palavras-chave: tratamento eletroquímico, eletrodos DSA, aço carbono, hidrogênio.

\section{ABSTRACT}

The electrochemical treatment as an alternative technological resource for the treatment of industrial effluents has attracted considerable attention through the last years because of important characteristics such as easy automation, high efficiency and versatility in a short period of time besides requiring less physical space for its units. As the electron is the main "reagent" in an electrochemical process, the use of chemicals is reduced. This and the possibility of using coproducts generated during the electrochemical treatment processes configure relevant opportunities in the actual global energy and sustainability scenario. These technologies may include the use of renewable energy sources such as Hydrogen, a cathodic coproduct of great ambiental and economical interest that could be obtained and stored. 
In this context, the selection of electrodes materials, especially those for anodic steps, are fundamental. The efficiency and sustainability of the electrochemical processes relies on materials with properties such as low electrochemical impedance and high corrosion resistance besides high electro activity for the oxidation of the compounds of interest.

The purpose of this article is to present some of the results of the electrochemical treatment developed by Labcorr aiming the removal of $\mathrm{NH}_{3}-\mathrm{N}$ and $\mathrm{COD}$ from industrial effluents. Electrochemical techniques were used for anodic and cathodic materials selection. The use of $\mathrm{NaOCl}$ as indirect oxidant was evaluated. Results indicated that a parallel plate reactor with DSA anodes and carbon steel cathodes and $\mathrm{NaOCl}$ injections at the beginning of the treatment process and in an intermediary point was an effective configuration for the removal of the compounds of interest. There is also a good indication of the possibility of nitrite and Hydrogen obtainment. The former could be used as corrosion inhibitor and the later as an energy source for the electrochemical treatment system in a semi-autonomous way.

Keywords: electrochemical treatment, DSA electrodes, carbon steel, hydrogen.

\section{INTRODUÇÃO}

O tratamento de efluentes não se configura apenas em uma questão de evitar impactos ambientais, mas é também um dispositivo para mitigação de problemas como corrosão e incrustação, onde o reuso é de grande interesse [1]. As indústrias, em específico as companhias de óleo e gás, são unidades bastante consumidoras de água e geradoras de efluentes [2-4]. Devido a essa característica, é de grande proveito a otimização do manejo destes efluentes, em especial, no que diz respeito ao reuso.

A aplicação do tratamento eletroquímico a efluentes industriais apresenta uma evolução relativamente recente, sendo bastante atrativa por apresentar características como facilidade de automação, maior eficiência e versatilidade em um menor tempo de tratamento, além de requerer menor espaço físico para suas instalações, permitindo operar com uma infraestrutura mais compacta [4-14]. Uma das maiores vantagens do tratamento eletroquímico é a diminuição no uso de produtos químicos para o tratamento [5, 9,13,15-17]. Outra importante vantagem é a possibilidade de uma eficiente mineralização de compostos orgânicos não biodegradáveis, além do alto potencial de desinfecção [7,16]. A combinação do tratamento eletroquímico a outras técnicas já existentes apresenta elevado potencial de aplicação [16-18]. A possibilidade de reaproveitamento de coprodutos gerados também é um grande atrativo deste processo.

Os processos eletroquímicos incluem técnicas como eletroflotação, eletrocoagulação, redução eletroquímica, Fenton, eletro-Fenton, eletrooxidação, entre outras, utilizadas isoladas ou combinadas a outras técnicas não eletroquímicas $[9,16,18-20]$. O foco deste trabalho é o método da eletrooxidação ou oxidação eletroquímica, que tem se mostrado efetivo na remoção de compostos orgânicos e inorgânicos [3,4,8,14,20-24].

Alguns parâmetros são críticos em um processo eletroquímico [4,7,8,14,16-18,25-30]. A densidade de corrente é provavelmente o parâmetro de maior influência - a ele estando diretamente relacionada a eficiência do tratamento em termos operacionais e de custos. O transporte de massa é outro fator crucial na remoção dos compostos desejados. A aeração do sistema, o aumento da vazão e a configuração adotada para o reator eletroquímico auxiliam no aumento do transporte de massa favorecendo a eficiência do processo [31]. Os materiais utilizados como eletrodos são de importância fundamental, também estando diretamente relacionados com a eficiência dos processos eletroquímicos e sua sustentabilidade. É necessária a utilização de materiais com propriedades especificas, como baixa impedância eletroquímica e elevada resistência à corrosão, além de elevada atividade catalítica para a oxidação dos compostos que se deseja remover. Metais e compostos como Ti, Pt, $\mathrm{PbO}_{2}$, DSA (ou ADE - anodo dimensionalmente estável) e BDD (Boron Dopped Diamond) têm sido utilizados nos estudos a respeito desta técnica. O custo também deve ser considerado neste contexto [3046].

Outro parâmetro relevante é a oxidação indireta - processo coadjuvante à oxidação direta que ocorre na superfície do anodo. Um composto de elevado poder oxidante (radical hidroxila, hipoclorito, entre outros), gerado pelo processo ou presente no meio, atua como auxiliar às reações de oxidação promovidas pelo anodo acelerando as reações de oxidação do meio aumentando assim a eficiência do tratamento eletroquímico [12,33,37,39,47-52]. É importante ressaltar que o efeito da oxidação indireta também dependerá do material selecionado para o anodo [52]. Além disso, poderá contribuir para a diminuição do valor de $\mathrm{pH}$ do meio ou ainda ser responsável por processos corrosivos significativos que comprometeriam o desempenho dos eletrodos no sistema eletroquímico $[8,39,47,51,53]$. No entanto, com um correto monitoramento do sistema de tratamento, é possível manter essas variáveis sob controle.

$\mathrm{O}$ trabalho aqui apresentado objetiva o uso do tratamento eletroquímico para a remoção de $\mathrm{N}_{-} \mathrm{NH}_{3} \mathrm{e}$ 
DQO. Efluentes contendo amônia e íons amônio e elevada demanda química de oxigênio apresentam potencial toxicidade podendo causar sérios danos ambientais se não tratados adequadamente antes de seu descarte.

A eletrooxidação do nitrogênio amoniacal $\left(\mathrm{N}-\mathrm{NH}_{3}\right)$ abrange algumas etapas e resulta na formação de diferentes compostos de maior estado de oxidação como nitrito e nitrato, além de nitrogênio [32,48], como indicado pela Equação 1.

$$
\begin{array}{cc}
\mathrm{NH}_{3} \leftrightarrow \mathrm{NH}_{2} \mathrm{OHad} \leftrightarrow \mathrm{NOHad} \leftrightarrow & \mathrm{NO} \leftrightarrow \mathrm{NO}_{2}^{-} \leftrightarrow \mathrm{NO}_{3}^{-} \\
& \downarrow \downarrow \\
& \mathrm{NH}_{2} \mathrm{~N}_{2} \mathrm{O} \leftrightarrow \mathrm{N}_{2}
\end{array}
$$

A seletividade destas reações dependerá do controle do sistema de tratamento eletroquímico, em especial do potencial e $\mathrm{pH}$ selecionados para o processo. A dependência do valor de $\mathrm{pH}$ é importante pois determina o mecanismo de decomposição das espécies nitrogenadas. Em meio aquoso, as espécies $\mathrm{NH}_{3} \mathrm{e}_{4}{ }^{+}$ estão em um equilíbrio controlado pelo pH, de acordo com a Equação 2.

$\mathrm{NH}_{4}^{+}+\mathrm{OH}^{-} \leftrightarrow \mathrm{NH}_{3}+\mathrm{H}_{2} \mathrm{O}$

$\mathrm{pKb}=4.74$

Em meio alcalino, com $\mathrm{pH}>10$, a espécie predominante é o $\mathrm{NH}_{3}$, enquanto a espécie $\mathrm{NH}_{4}{ }^{+}$predomina em $\mathrm{pH} \leq 7$. Para o valor intermediário, $7<\mathrm{pH}<10$, ambas as espécies coexistem em equilíbrio. Em meio alcalino, a oxidação ocorre através do mecanismo de adsorção da espécie $\mathrm{NH}_{3}$ na superfície do eletrodo e, em potencial mais elevado, a espécie é oxidada a $\mathrm{N}_{2}$ seguindo algumas etapas $[32,38,48] . \mathrm{Em} \mathrm{pH} \leq 7$, onde a espécie dominante é o íon $\mathrm{NH}_{4}{ }^{+}$, o mecanismo é a decomposição eletroquímica direta sem adsorção da espécie no eletrodo, formando $\mathrm{N} 2[32,48,49]$.

Um controle adequado é necessário uma vez que a possibilidade de formação de espécies intermediárias deve ser considerada e as espécies oxidadas incompletamente mais o radical $\mathrm{OH}^{-}$gerado durante o processo podem bloquear a superfície dos anodos, o que reduziria a eficiência do processo [32-37,48,50,53]. No entanto, a versatilidade do sistema eletroquímico permite atingir uma configuração em que a formação de espécies intermediárias e o bloqueio dos eletrodos seja bastante diminuído. O uso de oxidantes indiretos se configura em uma importante alternativa nesse contexto.

A geração de $\mathrm{N}_{2}$ e $\mathrm{H}_{2}$ são pontos notáveis do processo, sendo ambos os produtos bastante estáveis em uma extensa faixa de $\mathrm{pH}[33]$.

O objetivo deste trabalho é apresentar um processo de tratamento eletroquímico desenvolvido pelo Labcorr para remoção de $\mathrm{N}_{-} \mathrm{NH}_{3}$ e DQO. Este sistema de tratamento está em otimização visando a transposição para escala piloto.

\section{MATERIAIS E MÉTODOS}

A metodologia completa utilizada neste trabalho foi descrita em dois artigos publicados pelos autores [8,27].

A primeira etapa consistiu em uma avaliação, através das técnicas eletroquímicas de voltametria cíclica e polarização anódica, dos materiais passíveis de serem utilizados como anodos no processo de tratamento. Foram avaliados os materiais $\mathrm{Pt}, \mathrm{Ti} / \mathrm{RuO}_{2} \mathrm{DSA}{ }^{\circledR}, \mathrm{Ni}, \mathrm{Al}$ anodizado e grafite e dentre eles selecionado o que apresentou melhor desempenho para a oxidação eletroquímica do $\mathrm{N}_{-} \mathrm{NH}_{3}$ e resistência à corrosão provocada pelo meio. Para esta etapa, utilizou-se uma solução de $\left(\mathrm{NH}_{4}\right)_{2} \mathrm{SO}_{4}$ na concentração de 1000 ppm, com $\mathrm{pH}$ ajustado por solução de $\mathrm{NaOH} 1 \mathrm{M}$, simulando um efluente amoniacal não contendo cloretos. Baseado no equilíbrio indicado pela Equação (2), dois valores de pH foram considerados para os testes: 7 e 9,4. Foram utilizados o eletrodo de platina como contra eletrodo e o eletrodo de calomelano como referência.

Com o material do anodo devidamente selecionado, a etapa seguinte do trabalho foi de avaliação da configuração escolhida para o reator eletroquímico. Foi utilizado um reator de placas paralelas construído de material inerte e dois pares de eletrodos: dois anodos e dois catodos de mesmas dimensões. A distâncias entre os eletrodos foi de $10 \mathrm{~mm}$. A Figura 1 mostra o reator eletroquímico descrito e o posicionamento dos eletrodos. 


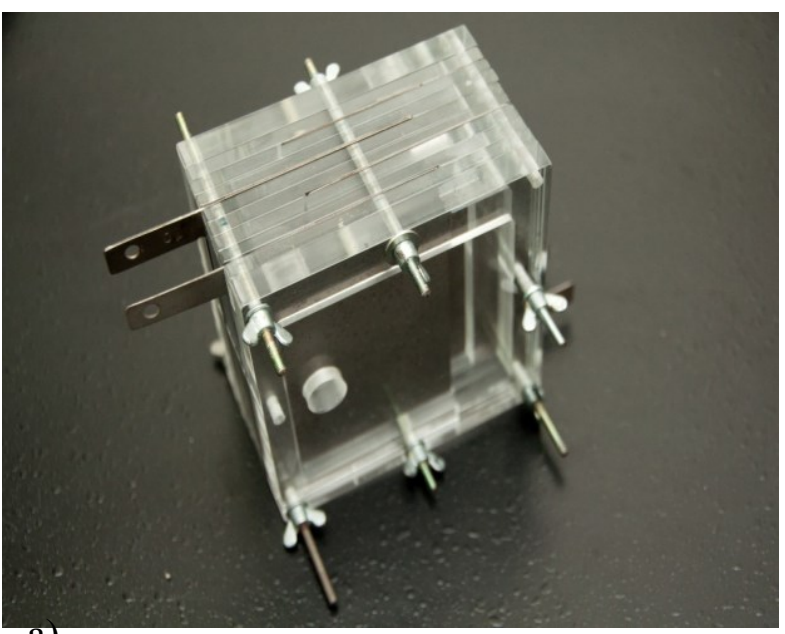

a)

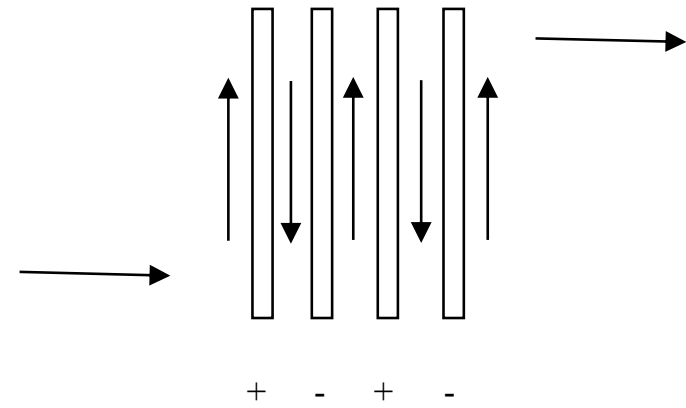

b)

Figura 1: a) Reator eletroquímico de placas paralelas; b) posição dos eletrodos de acordo com o fluxo da solução indicado pelas setas [8].

A maior eficiência apresentada por um reator de placas paralelas é reconhecida [31]. No entanto, um estudo hidrodinâmico foi feito para demonstrar a adequação desta configuração de reator para o sistema de tratamento eletroquímico proposto tendo sido determinado o valor do coeficiente de massa obtido. Após esta etapa, o reator foi então utilizado para o tratamento de um efluente industrial de uma refinaria de petróleo com elevados níveis de $\mathrm{N}-\mathrm{NH}_{3}$ e DQO. O tratamento foi realizado em batelada e à temperatura ambiente e pressão atmosférica. Como oxidante indireto, foi utilizado $\mathrm{NaOCl}$ em proporção baseada em estudos prévios e literatura $[29,32,35,39,48,51]$.

As análises químicas para determinação das concentrações das espécies de interesse foram realizadas utilizando metodologia definida por normas ASTM (American Society for Testing and Materials) e USEPA (United States Environmental Protection Agency) com auxílio de fotômetros da Hanna Instruments.

\section{RESULTADOS E DISCUSSÃO}

A seleção de materiais para utilização como anodos em um sistema de tratamento eletroquímico é uma etapa crucial pois a eficiência energética do processo está relacionada à atividade do material para a oxidação das espécies de interesse, o que também influenciará no custo total do tratamento. Neste contexto, Pt e os anodos dimensionalmente estáveis a partir de $\mathrm{Ti} / \mathrm{RuO}_{2}$ - DSA ${ }^{\circledR}$ têm sido amplamente utilizados em diferentes sistemas oxidativos. Além de elevada estabilidade e resistência a corrosão, esses materiais possuem elevada afinidade e atividade para processos oxidativos envolvendo espécies como o nitrogênio amoniacal, especialmente em meio alcalino [33]. Os testes com esses materiais objetivaram confirmar seu desempenho e gerar dados para a comparação com os outros materiais envolvidos nessa etapa, que foram escolhidos por se tratarem de alternativas interessantes sob o ponto de vista econômico sendo, no entanto, necessário estudar ainda sua viabilidade técnica. Os resultados são apresentados detalhadamente no artigo publicado pelos autores em [27].

Ni e Al anodizado não apresentaram características que os levassem à escolha para utilização como anodos no sistema de tratamento eletroquímico. Ambos apresentaram processos corrosivos em $\mathrm{pH}=7$. Os voltamogramas não indicaram atividade catalítica do $\mathrm{Al}$ anodizado para a oxidação das espécies amônia e íon amônio. Portanto, a utilização deste material foi descartada. O Ni, por sua vez, apresentou atividade para os processos oxidativos de interesse. Contudo, a resistência a corrosão é um fator importante pois nem o consumo do eletrodo e nem a contaminação do meio por íons $\mathrm{Ni}^{2+}$ são desejáveis. Este material também foi descartado para a etapa seguinte.

$\mathrm{O}$ grafite é um material termodinamicamente estável em soluções aquosas de $\mathrm{pH}<13$ [55]. Em teoria, o carbono poderia reagir tanto por oxidação gerando $\mathrm{CO}_{2}$, ácidos carbônicos e carbonatos quanto por redução, formando metano, álcool metílico e outros compostos orgânicos. Embora energeticamente possíveis, essas reações são altamente irreversíveis e não ocorrem sob temperatura ambiente e pressão atmosférica [55], 
condições deste estudo. No entanto, os resultados obtidos não foram conclusivos quanto a atividade catalítica deste material para a oxidação dos compostos amoniacais desejados. Os voltamogramas indicaram reações paralelas que precisariam ser melhor definidas através de um estudo eletroquímico mais específico. Assim, este material também não foi selecionado para a etapa seguinte, a de teste do reator eletroquímico.

Os testes realizados com os materiais $\mathrm{Ti} / \mathrm{RuO}_{2}-\mathrm{DSA} \AA$ e Pt confirmaram seu desempenho em relação à oxidação de compostos amoniacais. Os eletrodos DSA são mais efetivos do que os de Pt devido aos elementos estruturais adicionais como Ru, que apresentam elevada atividade para oxidação da amônia [54]. Através dos voltamogramas obtidos, observou-se que a Pt poderia promover a geração de produtos indesejáveis a partir da oxidação da amônia, como óxidos nitrogenados. $\mathrm{Em} \mathrm{pH}=7$, o voltamograma indicou a ocorrência de um pico catódico em aproximadamente $-0.12 \mathrm{~V}$. Nessas condições, a redução do $\mathrm{NO}_{2}{ }^{-}$a $\mathrm{N}_{2} \mathrm{O}$ é termodinamicamente possível, de acordo com a Equação 3 [32,48,49,55].

$$
\begin{aligned}
& 2 \mathrm{NO}_{2}^{-}+3 \mathrm{H}_{2} \mathrm{O}+4 e^{-} \rightarrow \mathrm{N}_{2} \mathrm{O}+6 \mathrm{OH}^{-} \\
& \mathrm{E}_{0}(\mathrm{SCE})=-0.092 \mathrm{~V}
\end{aligned}
$$

Este pico representa uma reação indesejada para o tratamento eletroquímico. No entanto, a versatilidade do sistema permite a modulação do mesmo para que não se atinja essa condição em específico, o que comprometeria a eficiência do processo. Esse comportamento catódico não foi observado para o eletrodo de $\mathrm{Ti} / \mathrm{RuO}_{2}$ - DSA ${ }^{\circledR}$. Portanto, após esta primeira etapa, foi possível selecionar o material $\mathrm{Ti} / \mathrm{RuO}_{2}$ - DSA ${ }^{\circledR}$ como o mais adequado para a utilização como anodo na configuração do reator eletroquímico.

A próxima etapa foi a avaliação da configuração de placas paralelas selecionada para o reator eletroquímico. Uma avaliação hidrodinâmica foi executada para a verificação da adequação do design de placas paralelas para o reator eletroquímico proposto. $\mathrm{O}$ valor do coeficiente de transferência de massa $\left(\mathrm{K}_{1}\right)$ foi calculado através da Equação 4, considerando um regime de fluxo turbulento.

$$
K_{1}=0,023 R e^{0,8} S c^{1 / 3} \mathrm{Di} / \mathrm{de}
$$

Onde:

$\mathbf{D i}=$ coeficiente de difusão do íon $\mathrm{NH}_{4}^{+}$(assumindo o controle mais lento do processo) $=1,957 \times 10^{-9} \mathrm{~m}^{2} \cdot \mathrm{s}^{-1}$ de $=$ diâmetro hidráulico $=9.41 \times 10^{-3} \mathrm{~m}$

$\mathbf{S c}=$ número de Schmidt $=\mu /(\rho \times D i) ; \mu=$ viscosidade da água $=1 \times 10^{-3}$ N.s.m ${ }^{-2}, \rho=$ densidade da água $=$ $1000 \mathrm{~kg} \cdot \mathrm{m}^{-3}$

$\mathbf{R e}=$ número de Reynolds $=($ de $\mathrm{x} \rho \mathrm{x} v) / \mu ; \mathrm{v}=$ velocidade do fluido $=\mathrm{Q} / \mathrm{A} \mathrm{m} \cdot \mathrm{s}^{-1}, \mathrm{Q}=$ velocidade do fluido $=8.5 \times 10^{-5} \mathrm{~m}^{3} \cdot \mathrm{s}^{-1}, \mathrm{~A}=$ área da passagem hidráulica do fluido $=4 \times 10^{-4} \mathrm{~m}^{2}$

O valor típico para o coeficiente de transferência de massa, considerando a aplicação industrial, situase entre $10^{-6}$ a $10^{-4} \mathrm{~m} \cdot \mathrm{s}^{-1}$, dependendo de fatores como custos de produção, manutenção e eficiência do processo [31]. De acordo com a Equação 4, o resultado obtido para $\mathrm{K}_{1}$ com a configuração proposta neste trabalho foi $K_{1}=1,64 \times 10^{-5} \mathrm{~m}_{\mathbf{s}} \mathbf{s}^{-1}$. Este valor está dentro da faixa considerada industrialmente, indicando que a configuração escolhida para o reator eletroquímico é adequada.

O sistema de tratamento eletroquímico foi então estabelecido, utilizando-se o reator de placas paralelas com anodos DSA e catodos de aço carbono. A Figura 2 indica um esquema simplificado do sistema, composto por um tanque de efluente e mistura, o reator eletroquímico com uma fonte de corrente, filtro e bomba para a recirculação. Os testes com este sistema foram realizados em batelada. 


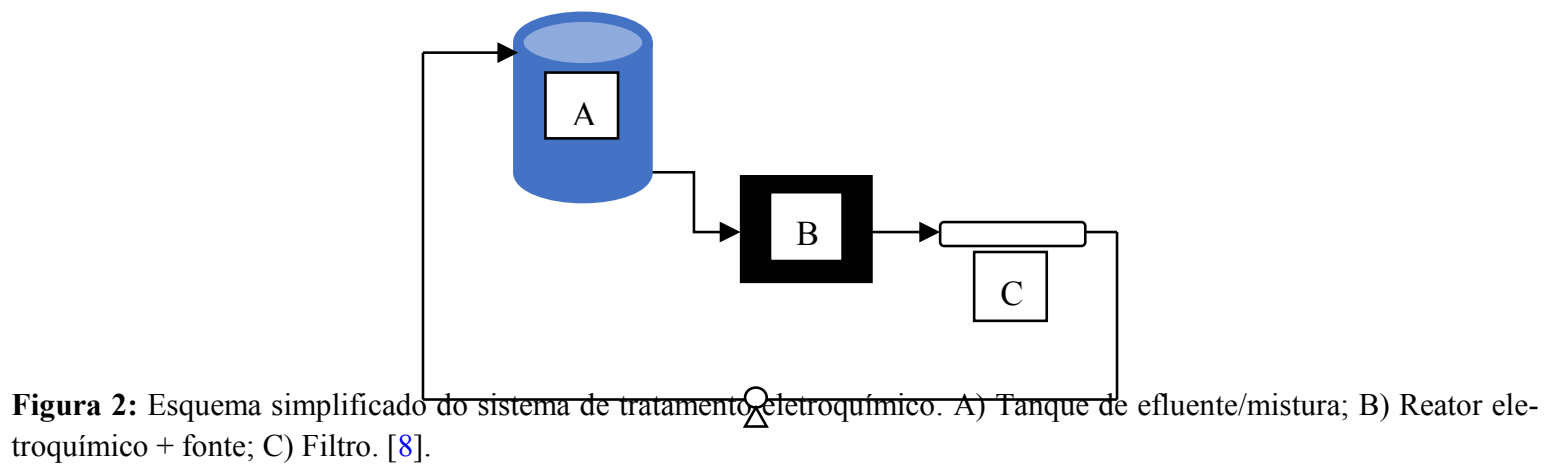

As amostras de efluente industrial de uma refinaria de petróleo foram então tratadas, verificando-se inclusive a resposta do processo de acordo com a otimização da injeção de oxidante indireto através da adição de $\mathrm{NaOCl}$ ao sistema. Os testes foram divididos em 3 categorias: Teste 1, com hipoclorito adicionado ao início do tratamento, Teste 2, com adição inicial e em 2 pontos intermediários e Teste 3, com adição inicial e em 1 ponto intermediário. Em todas as injeções de $\mathrm{NaOCl}$ utilizou-se o mesmo volume. As concentrações de $\mathrm{N}-\mathrm{NH}_{3}, \mathrm{NO}_{2}{ }^{-}, \mathrm{NO}_{3}{ }^{-}, \mathrm{DQO}$, cloraminas e Fe solúvel foram monitoradas com o tempo, bem como o valor de $\mathrm{pH}$. Essas medidas foram motivadas pelo caráter reversível de algumas reações, especialmente a formação de nitrito e nitrato, conforme indicado pela Equação 1. A quantidade de ferro solúvel foi medida como uma estimativa da estabilidade do catodo no meio de teste. Os resultados são apresentados detalhadamente em [8].

A formação de cloraminas durante o tratamento eletroquímico é possível quando há presença de cloretos no meio. Esse processo é dependente do valor de $\mathrm{pH}$, temperatura e da razão entre as concentrações de cloro ativo e amônia $[29,56]$. Alguns autores $[29,36,39,56]$ indicam que as cloraminas são instáveis e podem ser completamente extinguidas ao final do processo no catodo, sendo levadas a $\mathrm{N}_{2}$ através do adequado controle do sistema eletroquímico.

Os resultados obtidos com o tratamento eletroquímico indicaram uma forte tendência do comportamento do valor de $\mathrm{pH}$ em relação a quantidade de $\mathrm{NaOCl}$ utilizada. De uma maneira geral, quanto mais hipoclorito (Teste $1<$ Teste $3<$ Teste 2), maior o decaimento do valor de $\mathrm{pH}$, podendo tornar o efluente final impróprio para descarte de acordo com os parâmetros estabelecidos pela legislação vigente. Esse decaimento seria resultado das reações de hidrólise do cloro, passíveis de ocorrerem em meios contendo cloreto ou hipoclorito. A variação de $\mathrm{pH}$ influencia outros parâmetros também, como a quantidade de Fe solúvel no sistema, decorrente de processos de corrosão no catodo, e a formação de cloraminas. Embora as condições sejam termodinamicamente favoráveis à imunidade do $\mathrm{Fe}$, a variação de $\mathrm{pH}$ pode promover processos corrosivos no material. O perfil de concentração observado para o $\mathrm{Fe}$ indicou este comportamento, ou seja, quanto maior a quantidade de hipoclorito, menor o pH, maior a quantidade de Fe solúvel no meio. Em relação à cloramina, o menor valor de $\mathrm{pH}$ favoreceu a sua decomposição.

A formação de nitrito e nitrato também foi observada com o decaimento do nitrogênio amoniacal. Os perfis de concentração destes compostos durante o tratamento eletroquímico apresentaram características complementares, evidenciando o caráter reversível de formação mas tendendo à diminuição de concentração de todos, evidenciando a geração de nitrogênio e hidrogênio.

A variação da remoção do nitrogênio amoniacal e DQO pode ser observada pelos dados da Tabela 1 .

Tabela 1: Remoção de DQO com o tratamento eletroquímico.

\begin{tabular}{l|l|l}
\hline TESTE & $\begin{array}{l}\text { N-NH } \\
\text { REMOÇÃO \% }\end{array}$ & $\begin{array}{l}\text { DQO } \\
\text { REMOÇÃO \% }\end{array}$ \\
\hline 1 & 40,9 & 93,7 \\
\hline 2 & 85 & 74,6 \\
\hline 3 & 68,2 & 85,2 \\
\hline
\end{tabular}

Como pode ser observado, o decaimento do nitrogênio amoniacal foi obtido e mostrou ser proporcio- 
nal à quantidade de oxidante indireto no sistema, tendo chegado a $85 \%$ no caso do Teste 2 , onde 3 injeções de $\mathrm{NaOCl}$ ao total foram efetuadas. No entanto, esta condição apresentou a maior diminuição do valor de $\mathrm{pH}$, o que é indesejado conforme mencionado anteriormente. A diminuição da DQO e a variação deste parâmetro em relação a quantidade de oxidante indireto foram evidenciadas, sendo obtido a maior diminuição durante o Teste 1. A remoção eletroquímica dos compostos expressos pelo parâmetro DQO é favorecida por valores de $\mathrm{pH}$ mais alcalinos [57], o que seria o caso das condições do Teste 1 devido à menor quantidade de $\mathrm{NaOCl}$ durante o processo eletroquímico. Com estes resultados, as condições ótimas para o sistema de tratamento proposto foram alcançadas com o Teste 3 .

O tratamento eletroquímico apresentado neste trabalho apresenta percentuais promissores para a remoção de nitrogênio amoniacal e DQO, além de possibilidades interessantes de obtenção de coprodutos como nitrito (inibidor de corrosão) e hidrogênio (fonte de energia limpa).

\section{CONCLUSÕES}

A oxidação do $\mathrm{N}-\mathrm{NH}_{3}$ ocorre por diferentes mecanismos, dependentes do $\mathrm{pH}$ e consequentemente do equilíbrio $\mathrm{NH}_{3} / \mathrm{NH}_{4}^{+}$. Dentre os coprodutos gerados estão os compostos $\mathrm{NO}_{2}^{-}, \mathrm{NO}_{3}^{-}, \mathrm{N}_{2}$ e $\mathrm{H}_{2}$. A atividade eletroquímica dos eletrodos DSA ${ }^{\circledR}$ para oxidação do $\mathrm{N}-\mathrm{NH}_{3}$ foi confirmada bem como seu satisfatório comportamento face a processos corrosivos. Os outros materiais testados não se mostraram adequados para uma eficiente oxidação eletroquímica aliado à resistência à corrosão. Os parâmetros hidrodinâmicos obtidos sugerem a viabilidade de aplicação em escala industrial da configuração de placas paralelas para o reator eletroquímico.

$\mathrm{O} \mathrm{NaOCl}$ é um importante oxidante indireto havendo, contudo, um limite superior para a sua adição, mantendo ativo o processo de oxidação sem afetar de forma adversa o $\mathrm{pH}$ do meio. A possibilidade de remoção do $\mathrm{N}_{-} \mathrm{NH}_{3}$ e DQO foi confirmada. O aprimoramento da técnica permitirá gerar efluentes tratados com valores finais de concentração de contaminantes de acordo com os limites estabelecidos pela legislação ambiental vigente além do reaproveitamento de coprodutos como o $\mathrm{H}_{2}$, que poderá ser utilizado como fonte de energia para alimentar o próprio sistema de tratamento de forma semiautônoma.

\section{AGRADECIMENTOS}

Agradecemos ao auxílio financeiro concedido pelo CNPQ. Agradecemos também à Petrobras SA pelo fornecimento das amostras de efluente.

\section{BIBLIOGRAFIA}

[1] SOUZA, R.B.A., RUOTOLO, L.A.M., "Electrochemical treatment of oil refinery effluent using borondoped diamond anodes", Journal of Environmental Chemical Engineering, v.1, pp. 544-551, Sep. 2013.

[2] EL-NAAS, M.H., ALHAIJA, M.A., AL-ZUHAIR, S., "Evaluation of a three-step process for the treatment of petroleum refinery wastewater", Journal of Environmental Chemical Engineering, v. 2, pp. 56-62, Mar. 2014.

[3] COSTA, P.R.F., SILVA, D.R., MARTÍNEZ-HUITLE, C.A., et al., "Fuel station effluent treatment by electrochemical technology", Journal of Electroanalytical Chemistry, v. 763, pp. 97-103, Fev. 2016.

[4] SILVA, A.J.C., SANTOS, E.V., MORAIS, C.C.O., et al., "Electrochemical treatment of fresh, brine and saline produced water generated by petrochemical industry using $\mathrm{Ti} / \mathrm{IrO}_{2}-\mathrm{Ta}_{2} \mathrm{O}_{5}$ and $\mathrm{BDD}$ in flow reactor", Chemical Engineering Journal, v. 233, pp. 47-55, Nov. 2013.

[5] GHANBARI, F., MORADI, M. "A comparative study of electrocoagulation, electrochemical Fenton, electro-Fenton and peroxi-coagulation for decolorization of real textile wastewater: Electrical energy consumption and biodegradability improvement", Journal of Environmental Chemical Engineering, v. 3, pp. 499-506, Mar. 2015.

[6] THIRUGNANASAMBANDHAM, K., SIVAKUMAR, V., PRAKASH MARAN, J., "Response surface modelling and optimization of treatment of meat industry wastewater using electrochemical treatment", Journal of the Taiwan Institute of Chemical Engineers, v. 46, pp. 160-167. Jan. 2015.

[7] SANTOS, J. L. C., GERALDES, V., VELIZAROV, S., et al., "Characterization of fluid dynamics and mass-transfer in na electrochemical oxidation cell by experimental and CFD studies", Chemical Engineering Journal, v. 157, pp. 379-392, Mar. 2010.

[8] CANDIDO, L.C., GOMES, J. A. C. P., JAMBO, H. C. M., "Electrochemical treatment of Oil Refinery wastewater for NH3-N and COD removal", International Journal of Electrochemical Science, v. 8, pp. 9187 - 9200, Jul. 2013. 
[9] YAN, L., WANG, Y., LI, J., et al., "Comparative study of different electrochemical methods for petroleum refinery wastewater treatment", Desalination, v. 341, pp. 87-93, May 2014.

[10] JUNG, Y.J., BAEK, K.W., OH, B.S., et al., "An investigation of the formation of chlorate and perchlorate during electrolysis using $\mathrm{Pt} / \mathrm{Ti}$ electrodes: the effects of $\mathrm{pH}$ and reactive oxugen species and the results of kinetic studies", Water Research, v. 44, pp. 5345-5355, Oct. 2010.

[11] OZYONAR, F., KARAGOZOGLU, B. "Treatment of pretreated coke wastewater by electrocoagulation and electrochemical peroxidation processes", Separation and Purification Technology, v. 150, pp. 268-277, Aug. 2015.

[12] ROCHA, J.H.B., GOMES, M.M.S., FERNANDES, N.S., et al, "Application of electrochemical oxidation as alternative treatment of produced water generated by Brazilian petrochemical industry", Fuel Processing Technology, v. 96, pp. 80-87, Apr. 2012.

[13] RAMALHO, A.M.Z., MARTÍNEZ-HUITLE, C.A., SILVA, D.R., "Application of electrochemical technology for removing petroleum hydrocarbons from produced water using a DSA-type anode at different flow rates", Fuel, v. 89, pp. 531-534, Feb. 2010.

[14] JARRAH, N., MU'AZU, N.D. "Simultaneous electro-oxidation of phenol, $\mathrm{CN}^{-}, \mathrm{S}^{2-}$ and $\mathrm{NH}_{4}{ }^{+}$in synthetic wastewater using boron doped diamond anode", Journal of Environmental Chemical Engineering, v. 4, pp. 2656-2664, Sep. 2016.

[15] DUAN, F., LI, Y., CAO, H., WANG, Y., CRITTENDEN, J.C., et al., “Activated carbon electrodes: Electrochemical oxidation coupled with desalination for wastewater treatment", Chemosphere, v.125, pp. 205-211, Apr. 2015.

[16] ZHU, R., YANG, C., ZHOU, M., et al., "Industrial park wastewater deeply treated and reused by a novel electrochemical oxidation reactor", Chemical Engineering Journal, v. 260, pp. 427-433, Jan. 2015.

[17] PULKKA, S., MARTIKAINEN, M., BHATNAGAR, A., et al., "Electrochemical methods for the removal of anionic contaminants from water - A review", Separation and Purification Technology, v. 132, pp. 252-271, Aug. 2014.

[18] IGUNNU, E.T., CHEN, G.Z., "Produced water treatment technologies", International Journal of LowCarbon Technologies, v.0, pp. 1-21, Jul. 2012.

[19] ESTRADA-ARRIAGA, E.B., ZEPEDA-AVILES, J. A., GARCÍA-SÁNCHEZ, L., "Post-treatment of real oil refinery effluent with high concentrations of phenols using photo-ferrioxalate and Fenton's reactions with membrane process step", Chemical Engineering Journal, v. 285, pp. 508-516, Feb. 2016.

[20] LINARES-HERNÁNDEZ, I., BARRERA-DÍAZ, C., BILYEU, B., et al., "A combined electrocoagulation-electrooxidation treatment for industrial wastewater", Journal of Hazardous Materials, v. 175, pp. 688694, Mar. 2010.

[21] SANTOS, I. D., DEZOTTI, M., DUTRA, A. J. B., "Electrochemical treatment of effluents from petroleum industry using a Ti/RuO2 anode", Chemical Engineering Journal, v. 226, pp. 293-299, Jun. 2013.

[22] VALERO, D., GARCÍA-GARCÍA, V., EXPÓSITO, E., et al., "Electrochemical treatment of wastewater from almond industry using DSA-type anodes: direct connection to a PV generator", Separation and Purification Technology, v. 123, pp. 15-22, Feb. 2014.

[23] MATTIUSI, E.M., KAMINARI, N.M.S., PONTE, M.J.J.S., et al., "Behavior analysis of a porous bed electrochemical reactor the treatment of petrochemical industry wastewater contaminated by hydrogen sulfide $\left(\mathrm{H}_{2} \mathrm{~S}\right)$ ", Chemical Engineering Journal, v. 275, pp. 305-314. Sep. 2015.

[24] GARGOURI, B., GARGOURI O. D., GARGOURI, B., et al., "Application of electrochemical technology for removing petroleum hydrocarbons from produced water using lead dioxide and boron-doped diamond electrodes", Chemosphere, v. 117, pp. 309-315, Dec. 2014.

[25] SÄRKKÄ, H., BHATNAGAR, A., SILLANPÄÄ, M., "Recent developments of electro-oxidation in water treatment - A review", Journal of Electroanalytical Chemistry, v. 754, pp. 46-56, Oct. 2015.

[26] GHERNAOUT, D., NACEUR, M. W., AOUABED, A., "On the dependence of chlorine by-products generated species formation of the electrode material and applied charge during electrochemica water treatment”, Desalination, v. 270, pp. 9-22, Apr. 2011.

[27] CANDIDO, L.C., GOMES, J. A. C. P., "Evaluation of anode materials for the electro-oxidation of ammonia and ammonium ions", Material Chemistry and Physics, v. 129, pp. 1146-1151, May 2011. 
[28] MOOK, W.T., AROUA, M.K., ISSABAYEVA, G., "Prospective applications of renewable energy based electrochemical systems in wastewater treatment: A review", Renewable and Sustainable Energy Reviews, v. 38, pp. 36-46, Oct. 2014.

[29] GENDEL, Y., LAHAV, O., "Revealing the mechanism of indirect ammonia electrooxidation", Electrochimica Acta, v. 63, pp. 209-219, Feb. 2012.

[30] CHEN, J., SHI, H., LU, J., "Electrochemical treatment of ammonia in wastewater by RuO2-IrO2TiO2/Ti electrodes", Journal of Applied Electrochemistry, v.37, pp. 1137-1144, Jul. 2007.

[31] LIU, Y., LI, L., GOEL, R., "Kinetic study of electrolytic ammonia removal using $\mathrm{Ti}^{\mathrm{IrO}} \mathrm{I}_{2}$ as anode under different experimental conditions", Journal of Hazardous Materials, v. 167, pp. 959-965, Aug. 2009.

[32] KIM, K., KIM, Y., KIM, I., et al., "Electrochemical conversions characteristics of ammonia to nitrogen", Water Research, v. 40, pp. 1431-1441, Jan. 2006.

[33] VITSE, F., COOPER, M., BOTTE, G.G., "On the use of ammonia electrolysis for hidrogen production", Journal of Power Sources, v. 142, pp. 18-26, Dec. 2004.

[34] MARINCIC, L., LEITZ, F.B., "Electro-oxidation of ammonia in waste water", Journal of Applied Electrochemistry, v. 8, pp. 333-345, 1978.

[35] LIMA, R.M.G., WILDHAGEN, G.R.S., CUNHA, J.W.S.D., et al., "Removal of ammonium ion from produced waters in petroleum offshore exploitation by a batch single-stage electrolytic process", Journal of Hazardous Materials, v. 161, n. 2-3, pp. 1560-1564, Jan. 2009.

[36] LI, M., FENG, C., ZHANG, Z., et al., "Treatment of nitrate contaminated water using an electrochemical method", Bioresource Technology, v. 101, n. 16, pp. 6553-6557, Aug. 2010.

[37] KAPALKA, A., CALLY, A., NEODO, S., et al., "Electrochemical behavior of ammonia at $\mathrm{Ni} / \mathrm{Ni}(\mathrm{OH})_{2}$ electrode", Electrochemistry Communications, v. 12, n. 1, pp. 18-21, Jan. 2010.

[38] GERISCHER, H., MAUERER, A. "Untersuchungen zur anodischen oxidation von ammoniak an platinelektroden", Journal of Electroanalytical Chemistry and Interfacial Electrochemistry, v. 25, n. 3, pp. 421433, May 1970.

[39] LI, L., LIU, Y. “Ammonia removal in electrochemical oxidation: mechanism and pseudo-kinetics”, Journal of Hazardous Materials, v. 161, n. 2-3, pp. 1010-1016, Jan. 2009.

[40] GOOTZEN, J.F.E., WONDERS, A.H., VISSCHER, W., et al., "A DEMS and cyclic voltammetry study of $\mathrm{NH}_{3}$ oxidation on platinized platinum", Electrochimica Acta, v. 43, n. 12-13, pp. 1851-1861, May 1998.

[41] DELLA MONICA, M., AGOSTINIANO, A., CEGLIE, A. "An electrochemical sewage treatment process", Journal of Applied Electrochemistry, v. 10, pp. 527-533, 1980.

[42] WASMUS, S., VASINI, E.J., KRAUSA, M., et al., "DEMS-Cyclic voltammetry investigation of the electrochemistry of nitrogen compounds in $0.5 \mathrm{M}$ potassium hydroxide", Electrochimica Acta, v. 39, n. 1, pp. 23-31, Jan. 1994.

[43] SASAKI, K., HISATOMI, Y., "Oxidation and adsorption of ammonia on platinized platinum electrode", Journal of the Electrocheical. Society, v. 117, n. 6, pp. 758-762, 1970.

[44] FENG, C., SUGIURA, N., SHIMADA, S., et al., "Development of a high performance electrochemical wastewater treatment system", Journal of Hazardous Materials B, v. 103, pp. 65-78, Jul. 2003.

[45] ENDO, K., KATAYAMA, Y., MIURA, T., "Pt-Ir and Pt-Cu binary alloys as the electrocatalyst for ammonia oxidation", Electrochimica Acta, v. 49, pp. 1635-1638, Apr. 2004.

[46] YAO, K., CHENG, Y.F. "Fabrication by electrolytic deposition of Pt-Ni electrocatalyst for oxidation of ammonia in alcaline solution", International Journal of Hydrogen Energy, v. 33, n. 22, pp. 6681-6686, Nov. 2008.

[47] MA, X., WANG, R., GUO, W., et al., "Electrochemical removal of ammonia in coking wastewater using $\mathrm{Ti} / \mathrm{SnO}_{2}+\mathrm{Sb} / \mathrm{PbO}_{2}$ anode", Internationa Journal of Electrochemical Science, v. 7, pp. 6012-6024, Jul. 2012.

[48] KIM, K., KIM, Y., KIM, I., et al., "The electrolytic decomposition mechanism of ammonia to nitrogen at na IrO2 anode", Electrochimica Acta, v. 50, pp. 4356-4364, Mar. 2005.

[49] BARD, A.J., PARSONS, R., JORDAN, J. Standard Potentials in Aqueous Solution, New York,Marcel Dekker Inc., 1985. 
[50] BONNIN, E.P., BIDDINGER, E.J., BOTTE, G.G. "Effect of catalyst on electrolysis of ammonia effluents", Journal of Power Sources, v. 182, n. 1, pp. 284-290, Jul. 2008.

[51] SZPYRKOWICZ, L., KAUL, S.N., NETI, R.N., et al., "Influence of anode material on electrochemical oxidation for the treatment of tannery wastewater", Water Research,, v. 39, pp. 1601-1613, Mar. 2005.

[52] SCIALDONE, O., RANDAZZO, S., GALIA, A., et al., "Electrochemical oxidation of organics in water: role of operative parameters in the absence and in the presence of $\mathrm{NaCl}^{\prime}$, Water Research, v. 43, n. 8, pp. 2260-2272, May 2009.

[53] PANIZZA, M., BOCCA, C., CERISOLA, G., "Electrochemical treatment of wastewater containing polyaromatic organic pollutants", Water Research, v. 34, n. 9. Pp 2601-2605, Jun. 2000.

[54] DE VOOYS, A.C.A, KOPER, M.T.M., VAN SANTEN, R.A., et al., "The role of adsorbates in the electrochemical oxidation of ammonia on noble and transition metal electrodes", Journal of Electroanalytical Chemistry, v. 506, pp. 127-137, Mar. 2001.

[55] POURBAIX, M., Atlas of Electrochemical Equilibria in Aqueous Solutions, 2ed., Houston, National Association of Corrosion Engineers, 1974.

[56] KAPALKA, A., KATSAOUNIS, A., MICHELS, N., et al., "Ammonia oxidation to nitrogen mediated by electrogenerated active chlorine on $\mathrm{Ti} / \mathrm{PtO}_{\mathrm{x}}-\mathrm{IrO}_{2}$ ", Electrochemistry Communications, v. 12, n. 9, pp. 1203-1205, Sep. 2010.

[57] YEON, K-H., SONG, J-H., SHIM, J., et al., "Integrating electrochemical processes with electrodialysis reversal and electro-oxidation to minimize COD and T-N at wastewater treatment facilities of power plants", Desalination, v. 202, n. 1-3, pp. 400-410, Jan. 2007. 